\title{
Capacitação no uso das bases Medline e Lilacs: avaliação de conteúdo, estrutura e metodologia ${ }^{*}$
}

\author{
Angela Maria Belloni Cuenca \\ Maria do Carmo Avamilano \\ Alvarez

Maria Lucia Evangelista de
Faria Ferraz
Eidi Raquel Franco Abdalla \\ Eidi Raquel Franco Abdalla
}

\section{INTRODUÇÃO}

Com as novas tecnologias de acesso à informação, as bibliotecas, principalmente as acadêmicas, tiveram de introduzir em seus serviços a capacitação de usuários, para que essas tecnologias fossem rapidamente aceitas e utilizadas. Nos países avançados, várias bibliotecas, principalmente na área de medicina ${ }^{2,14}$, passaram a oferecer cursos específicos para que os usuários conhecessem os sistemas automatizados de recuperação da informação.

A descrição de treinamentos de usuários, necessidade e pertinência dos mesmos, bem como o impacto de uso de bases de dados para a recuperação da informação têm merecido atenção na literatura internacional. Sollenberger e Smith ${ }^{17}$ (1987) enfatizaram a importância de treinamentos em sala de aula, ou seja, a prática monitorada e acompanhada de aulas teóricas. Poisson ${ }^{13}$ (1986) foi além, ressaltando a importância do conhecimento sobre o vocabulário controlado específico de cada base para uma busca pertinente. Starr e Renford ${ }^{18}$ (1987) observaram que o aspecto mais positivo dos cursos foi dar oportunidade para o usuário conhecer os recursos de busca, por meio de descritores e qualificadores, dos vocabulários controlados, praticamente desconhecidos da maioria deles. Mueller e Foreman ${ }^{11}$, em 1987, já apresentavam dados sobre minicurso de acesso à base de dados Medline, desenvolvi-

\footnotetext{
* Avaliação realizada como parte da dissertação de mestrado "Usuário da Busca Informatizada: Avaliação do Curso Medline/Lilacs no Contexto Acadêmico", defendida na Faculdade de Saúde Pública da USP, em 1997.
}

do pela Medical School of the University of Minnesota. Hepworth ${ }^{7}$ (1991) apresentou critérios para estabelecer treinamento ao usuário final e sugeriu conteúdo para curso de capacitação da equipe de bibliotecários. Fisher e Bjorner ${ }^{6}$ (1994) discutiram o que deve ser ensinado ao usuário final em um ambiente eletrônico. Maxymuk ${ }^{10}$ (1991) descreveu treinamento para usuários finais em níveis diferenciados de acordo com a necessidade do usuário, ou seja, níveis básicos de treinamento e níveis avançados, utilizando diversas bases de dados com softwares diferentes.

Já no Brasil, há pouco registro de avaliação ou impacto de treinamentos para usuários, mesmo sendo conhecidas algumas experiências de bibliotecas acadêmicas investindo na capacitação de usuários para os seus catálogos online. $\mathrm{Na}$ área da saúde, Cuenca ${ }^{4}$, em 1997, avaliou um curso modulado para acesso às bases de dados Medline $e$ Lilacs, ministrado em biblioteca acadêmica.

\section{A Experiência da Biblioteca/CIR}

A Biblioteca: Centro de Informação e Referência em Saúde Púbica (CIR), da Faculdade de Saúde Pública da Universidade de São Paulo (FSP/USP), promove, desde 1993, cursos para capacitação de usuários para uso de sua rede local, principalmente para o acesso às bases de dados internacionais. Esses cursos fazem parte de seu Programa Educativo.

Um deles é o Curso Avançado de Acesso às Bases de Dados em cd-rom 
Medline e Lilacs, destinado principalmente aos docentes e alunos da pósgraduação da FSP/USP. Essas categorias de usuários, por força de sua necessidade acadêmica e de pesquisa, são as que mais precisam manterse a par da literatura que está sendo publicada nas suas áreas. Os cursos de pós-graduação na FSP detêm cerca de $70 \%$ do total de seus alunos.

A escolha da base bibliográfica Medline para essa capacitação deveu-se a sua abrangência internacional na área da saúde e também da base de dados Lilacs, porque divulga a literatura latino-americana nessa mesma área, incluindo, portanto, a nacional. Ambas indexam os principais periódicos necessários para o desenvolvimento da pesquisa na área da saúde. Outros motivos da escolha foram os seguintes: são as bases mais utilizadas pelos usuários da biblioteca, e, embora possuam interfaces de busca diferentes, estas são comuns a outras bases disponíveis na rede local.

Foram estabelecidas as seguintes metas para o curso: conseguir que o usuário elaborasse sua estratégia de busca utilizando vocabulário Medical Subject Headings (MeSH* ${ }^{*}$ e o Descritores em Ciências da Saúde $\left(\mathrm{DeCS}^{* *}\right)$; tornar o usuário familiarizado com as diferentes interfaces de busca das bases de dados; levar ao conhecimento do usuário os recursos disponíveis para obtenção de cópias de documentos por sistema em rede e online, além do tradicional.

O curso tem sido ministrado por bibliotecários experientes nos serviços de referência, de recuperação automatizada da informação e familiarizados com os temas da área da saúde. O domínio da terminologia em ciências da saúde, conhecimento das tecnologias para o acesso às bases e os vários softwares disponíveis em rede são condições básicas para o bibliotecário tornar-se instrutor dos cursos.

Dividido em dois módulos de quatro horas cada, o instrutor apresenta, no

\footnotetext{
* MeSH - vocabulário controlado utilizado na base de dados Medline.

** DeCS - vocabulário controlado, baseado no Mesh, utilizado na base de dados Lilacs.
}

primeiro, a base de dados Medline, seu histórico, estrutura e conteúdo, bem como seus campos e comandos para busca. Apresenta a lógica booleana e, como exercício prático, formula buscas simples (por palavras) com o usuário. Após esclarecimentos de dúvidas, são apresentadas informações sobre o $\mathrm{MeSH}$, descritores principais e secundários, além de respectivos qualificadores. Nessa oportunidade, novamente são colocados exercícios com estratégia de busca mais elaborada, para que os usuários possam elucidar as dificuldades encontradas.

No segundo módulo é apresentada a base de dados Lilacs, com os mesmos tópicos do Medline, histórico, conteúdo, comandos, campos disponíveis para a busca, funções e DeCS. O usuário então procede à busca em ambas as bases, no assunto de seu interesse, sob supervisão do bibliotecário, gravando seus resultados em disquetes. Ainda no segundo módulo, os alunos são orientados em como localizar os documentos selecionados, por meio de bases de localização*, e como obtêlos, via serviços de comutação bibliográfica.

O material didático distribuído durante o curso é preparado pela equipe de bibliotecários-instrutores e com a seguinte composição: programa do curso; apostila contendo os passos básicos e principais telas e exercícios; um guia básico dos principais campos de busca, operadores da base Medline e um cartão ou folha índice com o resumo dos comandos e funções do software (esses dois são fornecidos pelo fabricante); regulamento da Biblioteca para o uso das bases de dados e folders sobre os serviços da biblioteca.

O curso é ministrado com aulas teóricas e práticas nos próprios computadores da rede local da biblioteca. O

* São utilizadas: Dedalus (Banco de Dados Bibliográficos da USP), CCN-IBICT (Catálogo Coletivo Nacional do Instituto Brasileiro de Informação em Ciência e Tecnologia), Unibibli (CDROM com os acervos da USP, Unesp e Unicamp), SeCS (Seriados em Ciências da Saúde da Bireme). aluno é monitorado individualmente, durante todo o curso, quanto à realização dos exercícios propostos e, ao final, realiza levantamento retrospectivo na sua área temática. Os meios de ensino, além do quadro magnético, são o uso de transparências e do material didático para acompanhamento das aulas.

O curso tem sua divulgação restrita à comunidade da FSP, realizada por meio de carta-circular enviada aos docentes da FSP, bem como cartazes, afixados na área de leitura, balcão de empréstimo e nos murais da biblioteca. A divulgação externa é realizada por meio dos folders sobre os serviços que presta.

Essa experiência foi avaliada após dois anos de sua implantação (1993 a 1995) para que seus resultados contribuíssem para o aprimoramento do curso.

\section{OBJETIVO}

Este artigo tem como objetivo divulgar os resultados da avaliação do Curso Medline/Lilacs da Biblioteca/CIR da FSP/USP, quanto ao seu conteúdo, estrutura e metodologia, bem como as recomendações para o seu aprimoramento.

\section{MÉTODO}

A população para o estudo foi composta de 140 participantes dos 29 cursos Medline e Lilacs, ministrados entre novembro/1993 (primeiro curso) e novembro/1995 e assim distribuídos: 115 alunos da pós-graduação, 14 docentes da FSP/USP, além de 11 usuários externos envolvidos nas atividades acadêmicas e de pesquisa na área da saúde, portanto usuários necessitados de atualização constante da produção científica na área da saúde.

Utilizaram-se os dados coletados da segunda parte do questionário da avaliação geral realizada por Cuenca ${ }^{4}$, em 1997. Esse questionário, composto de questões abertas e fechadas, de múltipla escolha, na sua parte II, abordava aspectos sobre a estrutura, conteúdo e metodologia do Curso Medline e Lilacs, bem como solicitava ao egresso opiniões sobre conhecimentos prévios para melhor acompanhar o programa, sobre a suficiência do conteúdo, carga horá- 
ria, metodologia didática adotada, material instrucional distribuído e divulgação do curso. Outras opiniões nesse sentido também foram coletadas da última parte do referido questionário, no espaço livre para comentários.

Optou-se pelo uso de questionário, e não por entrevista, pela dificuldade em contactar os participantes, uma vez que a maioria deles era constituída de alunos da pós-graduação, residindo em diferentes localidades, com freqüência inconstante à FSP/USP.

\section{RESULTADOS E COMENTÁRIOS}

Dos 140 questionários enviados foram devolvidos 92 , correspondendo a $66 \%$ de retorno, no prazo estabelecido, sendo este, portanto, o universo estudado. As opiniões foram as seguintes:

\section{Quanto ao conteúdo programático:}

As informações sobre a Base de Dados Medline foram consideradas "suficientes" por 75\% (69) dos usuários e "insuficientes" por 25\% (23). Nenhum aluno considerou "excessivas" as informações recebidas. A "insuficiência" de informações foi devida, principalmente, à falta de maior tempo com os exercícios práticos junto aos computadores (18 alunos), considerados importantes na assimilação das informações dadas no curso.

Os vários trabalhos realizados sobre capacitação de usuários no acesso a bases de dados em cd-rom ${ }^{1,2,7,6,10,16} \mathrm{e}$, especificamente, sobre a base Medline, ${ }^{10,11}$, permitiram chegar a um núcleo básico de informações para o "conteúdo" da capacitação do usuário final. Assim, são destacados: uso de comandos e funções do software, campos disponíveis para a busca, formulação de estratégias de busca via descritores e termos livres, uso dos operadores lógicos booleanos como recursos fundamentais para uma boa estratégia e estrutura da base de dados. No caso do Curso Medline/Lilacs estes tópicos estiveram presentes, não precisando de adequações de conteúdo. Apenas devem ser sanados os motivos apontados como insuficientes.

O conteúdo para a capacitação no uso da Base de Dados Lilacs recebeu

TABELA 1

Opinião dos participantes sobre a utilidade do módulo sobre comutação bibliográfica no curso

\begin{tabular}{lcr}
\hline GRAU DE UTILIDADE & No & $\%$ \\
\hline Muito útil & 46 & 50,0 \\
Útil & 42 & 45,6 \\
Pouco útil & 3 & 3,3 \\
Insuficiente & 1 & 1,1
\end{tabular}

Indiferente

TOTAL

92

$100,0 \%$

qualificação semelhante à base Medline, ou seja, 77,2\% (71) dos usuários consideraram "suficientes" as informações recebidas, e 22,8\% (21), "insuficientes". Nenhum deles considerou "excessivas" as informações recebidas. Segundo a opinião daqueles que consideraram "insuficientes" as informações recebidas dessa base, a "falta de tempo com os exercícios práticos" novamente apareceu como a principal causa $(81 \%)$.

De fato, para essa base, em comparação com o oferecido para a base Medline, o curso disponibilizou menor número de horas, no módulo II, onde a Lilacs foi oferecida, o período de quatro horas foi dividido com as informações sobre a comutação bibliográfica. Este é um aspecto que deve ser revisto e aperfeiçoado quando da revisão do conteúdo do curso.

O módulo sobre como localizar e obter o documento selecionado, denominado Comutação Bibliográfica, foi incluído no curso para que os alunos pudessem dar seqüência à recuperação da informação. Segundo informação dos bibliotecários-instrutores, este módulo teve boa aceitação, sendo considerado, pelos egressos dos cursos, tão importante quanto os módulos sobre as bases de dados.

Esta informação confirma-se, conforme mostra, a seguir, a tabela 1 , quando $95,6 \%$ dos usuários o consideraram "muito útil" e "útil". Os alunos que o consideraram "pouco útil" (3,3\%) responderam que não tiveram este módu- lo cumprido no programa, o que, de certa forma, invalida esse percentual. A inserção desse tipo de informação em cursos para capacitação no acesso a bases de dados é de grande valia, já que, tão importante quanto saber da existência de uma informação, é saber como obtê-la. Nesse sentido, Maxymuk $^{10}$ (1991), em suas conclusões sobre instrução no uso de cd-roms, coloca que, em serviços de acesso a bases de dados, é essencial o fornecimento de cópias dos documentos, e não somente de suas citações.

Quanto ao cumprimento do conteúdo proposto, observou-se que algumas turmas do curso não tiveram todo o programa proposto. O "não-cumprimento do programa" foi sentido por $20 \%$ dos alunos quanto aos itens: comutação bibliográfica (14,1\%), na localização do documento $(3,3 \%)$ e na busca com o tema do aluno (2,2\%). Sobre este aspecto, cabe esclarecer que, devido à falta de salas exclusivas para os cursos, estes sofreram várias alterações de conteúdo e calendário, o que acabou prejudicando a estrutura proposta. Nesse sentido, Ring e Meer ${ }^{15}$ (1994) apresentaram desenho detalhado de uma sala para cursos dentro de uma biblioteca, contemplando aspectos como disposição da mobília, computadores, climatização, instalação elétrica, acústica, escolha de software, hardware, entre outros.

Pode-se considerar que, quanto ao seu núcleo básico, o programa desenvolvido para a capacitação do usuário final está adequado. No entanto, como su- 
gestão ao seu aprimoramento, outros tópicos poderiam complementar seu conteúdo, como: a) leituras prévias, monitoradas, sobre sistemas de informação e vocabulários especializados na área da saúde; b) informações sobre os serviços especializados prestados por bibliotecas e sistemas de informação; c) informações sobre outras bases de dados relevantes na área da saúde; d) maior detalhamento sobre a abrangência, origem e histórico da base Lilacs; e) maior detalhamento no uso dos softwares de cada base disponível na rede local; f) ênfase à necessidade de consulta ao manual de instruções, tanto o impresso quanto o disponível no próprio cd-rom; g) orientação sobre quando utilizar uma base de dados bibliográfica ou outra fonte mais adequada ao seu assunto.

\section{Quanto à estrutura do curso:}

As sugestões dos participantes do curso quanto à estrutura proposta, como mostra a tabela 2, concentraramse no aumento de aulas práticas para reforçar o conteúdo (68,5\%). Este fato confirma um dos motivos de insuficiência indicados no conteúdo programático.

Um curso exclusivo para a base de dados Lilacs mereceria maior atenção por parte da Biblioteca, pelos motivos: a) trata-se da única base de ciências da saúde a indexar trabalhos da literatura nacional (cerca de 180 periódicos brasileiros) e latino-americana, pouco divulgados nas bibliografias e bases de dados internacionais; b) a literatura nacional e latino-americana na área da saúde pública, bastante contemplada por essa base, é fundamental para o desenvolvimento de estudos e pesquisas nos países de Terceiro Mundo.

Sobre estrutura de cursos dessa natureza, encontram-se, na literatura internacional, várias experiências em bibliotecas acadêmicas ${ }^{7-11,14,17}$ que podem dar subsídios àquelas que estejam preparando seus treinamentos. Hepworth ${ }^{7}$ (1991), por exemplo, apresenta os tópicos principais que devem anteceder a estrutura do treinamento para recu-

TABELA 2

\section{Sugestões dos participantes para a adequação da estrutura do curso}

\begin{tabular}{lcc}
\hline SUGESTÕES* & № (T=92) & $\%$ \\
\hline $\begin{array}{l}\text { Colocaria mais aulas práticas para reforçar o } \\
\text { conteúdo }\end{array}$ & 63 & 68,5 \\
Dividiria em outros dois cursos & 15 & 16,3 \\
Acrescentaria módulo sobre como usar outras bases & 12 & 13,0 \\
$\begin{array}{l}\text { Outras: } \\
\text { Acrescentaria como usar Internet }\end{array}$ & 2 & 2,2 \\
Antes do curso, trabalharia somente com descritores & 1 & 1,1 \\
Nada mudaria & 8 & 8,7 \\
\hline
\end{tabular}

* Múltipla escolha por 92 usuários.

TABELA 3

Opinião quanto aos conhecimentos prévios necessários para a participação no curso

\begin{tabular}{lcc}
\hline CONHECIMENTO PRÉVIO* & № (T=92) & $\%$ \\
\hline Básico de informática & 65 & 71,5 \\
De inglês & 40 & 43,5 \\
De inglês e informática & 31 & 33,7 \\
Dos descritores principais da pesquisa & 22 & 23,9 \\
De uso das bases de dados & 9 & 9,8 \\
De nenhum dos indicados & 7 & 7,6 \\
De todos os indicados & 4 & 4,3 \\
Sem resposta & 1 & 1,1 \\
\hline
\end{tabular}

* Múltipla escolha por 92 usuários.

peração automatizada em sistemas de informação, na área da saúde. São eles: a) estabelecer a necessidade de treinamento; b) estabelecer os objetivos do treinamento; c) definir estratégias para viabilizá-lo; d) estimar os recursos necessários por meio de curso piloto; e) produzir recursos bibliográficos e didáticos apropriados; f) estabelecer avaliação e acompanhamento dos resultados.

Uma das dificuldades apontadas pelos bibliotecários instrutores do Medline/ Lilacs foi a heterogeneidade dos participantes, principalmente quanto aos conhecimentos prévios de informática e de idiomas. Isto se confirmou quando se perguntou aos participantes do curso sobre que conhecimentos prévios deveria ter o aluno para melhor acompanhá-lo. A maioria das respostas recaiu nos conhecimentos de informática básica $(70,6 \%)$ e de inglês $(43,5 \%)$ (tabela 3$)$.

O curso é programado para ter duração de oito horas, incluindo a prática nos computadores da rede. Essa carga horária foi considerada inadequada por $63 \%$ e adequada por $37 \%$ dos participantes. As respostas dos 58 participantes que consideraram a carga horária inadequada (tabela 4), apontam para uma estrutura ideal com 9 a 


\section{2 horas $(70,7 \%)$ de curso.}

$\mathrm{Na}$ literatura, descreveram-se cursos para capacitação no acesso a bases de dados com duração variada, de uma hora a até 20 horas (Nesbit e Miner, citados por Sollenberger e Smith ${ }^{17}$ ), nos quais a carga horária fica atrelada à especialização da turma, ao conhecimento prévio do aluno e à finalidade da capacitação. Ludwig ${ }^{9}$ (1988), por exemplo, descreveu treinamento de duas horas para que alunos de cursos de medicina pudessem ter acesso a uma base Medis, que representa um catálogo on-line acrescido de subconjunto da base Medline. Já Sollenberger e Smith ${ }^{17}$ (1987) descreveram curso para treinamento somente na base Medline com o mínimo de 8 horas, podendo ser prolongado para até 12 horas, dependendo da categoria do usuário. Mularski e Bradigan ${ }^{12}$ (1993) expuseram treinamento de 12 horas, distribuídas em períodos de três horas semanais, durante quatro semanas, somente para o uso da base Medline.

O Curso Medline e Lilacs, estruturado como está, deve ter sua carga horária revista e aumentada, para até 12 horas, na dependência da finalidade da capacitação e do conhecimento prévio da turma. Como sugestão, apontou um dos participantes que exercícios monitorados devem ser complementares ao curso, independentemente da carga horária do mesmo.

\section{Quanto à metodologia didática:}

A metodologia de ensino adotada, com aulas teóricas e práticas monitoradas individualmente, foi considerada adequada por $89,1 \%$ dos participantes, pouco adequada, por $7,6 \%$ deles, e inadequada, por $3,3 \%$.

Entre as sugestões dos que não a consideraram adequada, apontou-se necessidade de haver mais prática com o aluno, monitorada pelo bibliotecário instrutor, principalmente no uso do thesaurus. Nesse aspecto, os instrutores colocaram que algumas turmas, principalmente as formadas com alunos com menos experiência no uso da informação, precisariam de mais exem-

TABELA 4

\section{Sugestões dos participantes para adequação da carga horária para 0 curso}

\begin{tabular}{lcc}
\hline SUGESTÕES & № & $\%$ \\
\hline De 2 a 4 horas seria suficiente & 1 & 1,7 \\
De 4 a 6 horas seria suficiente & 1 & 1,7 \\
De 9 a 12 horas seria adequada & 41 & 70,7 \\
Mais que 12 horas & 15 & 25,9 \\
TOTAL & 58 & 100,0
\end{tabular}

plos práticos para fixação do conteúdo.

Dos 92 participantes, $84,8 \%$ consideraram suficiente o material didático distribuído durante o curso e 15,2\% "insuficiente". O material não foi considerado excessivo por nenhum deles.

Os comentários sobre a "insuficiência" do material didático distribuído foram dirigidos apenas às apostilas. Assim, sugeriu-se que as mesmas deveriam ser mais objetivas; com mais detalhamento sobre a base Lilacs; que apresentassem cópias das principais telas, que incluíssem exercícios resolvidos passo a passo, e, finalmente, que houvesse uma versão delas em vídeo.

O guia básico e resumo dos comandos e os folders devem ser mantidos para fins de orientação e divulgação. A biblioteca poderia também disponibilizar fitas de vídeo sobre as bases de dados e editar uma sobre sua rede local onde o aluno egresso pudesse sedimentar seu conhecimento, como os apresentados no trabalho de Reese ${ }^{14}$ (1993).

Deve ser destacado o importante papel dos instrutores que, além da habilidade de ensinar, devem possuir conhecimentos específicos da terminologia em ciências da saúde, das tecnologias de informática e dos vários softwaresdisponíveis.

Earl $^{5}$ (1991) apresentou experiência de uma biblioteca da área médica que in- vestiu no aluno de graduação para torná-lo multiplicador dos cursos para uso do Medline aos seus colegas. Uma vantagem foi que, como todos tinham interesse na mesma área, facilitou-se o entendimento entre instrutor e aluno. Outra vantagem foi o fato de o aluno ficar mais à vontade tirando dúvidas com o colega, do que com o bibliotecário. A desvantagem ficou por conta da informação incompleta que o alunoinstrutor tem sobre os termos do MeSH, uso dos operadores lógicos e estruturação de estratégias de busca. Nesse caso, o bibliotecário tornou-se o coordenador dos cursos e o formador de novos instrutores.

Um aspecto interessante, porém pouco evidenciado na implantação de cursos dessa natureza, foi o abordado por Sollenberger e Smith ${ }^{17}$ (1987), que calcularam o tempo gasto pelo instrutor na produção de um curso para busca informatizada, em biblioteca, onde foram gastas 200 horas do profissional bibliotecário e 100 horas do pessoal auxiliar, principalmente na adaptação do conteúdo às características da comunidade, na programação e divulgação, na configuração dos equipamentos, no preparo do material didático e exercícios, no curso-piloto e no preparo de leituras como reforço adicional ao conteúdo básico do curso.

Os bibliotecários de referência da Biblioteca da FSP, atuando como instrutores, realizaram um autotreinamento, repassando o conhecimento adquirido aos outros colegas e usuários. Este aspecto é relatado por Crellin ${ }^{3}$ (1992), em artigo sobre o impacto do uso do cd-rom nos serviços de referência, onde todo o corpo bibliotecário passou a ser treinado no uso dos CDs, inclusive com 


\section{atualização contínua.}

Outro aspecto de importância apontado pelos bibliotecários instrutores do curso foi a necessidade de adequação da infra-estrutura da biblioteca para resolver problemas relacionados com espaço físico específico para o curso, técnico de rede informatizada, microcomputadores suficientes para o número de alunos, bem como, recursos humanos com qualificação suficiente para manutenção do curso. Nesse sentido, após implantado o curso, há necessidade de atualização do conteúdo de acordo com os recursos dos novos softwares, das apostilas, material audiovisual, divulgação, preparo antecipado da estratégia de busca de acordo com o perfil do aluno participante, organização das turmas de acordo com a disponibilidade de instrutores e necessidade do usuário, assim como adequação dos horários dos instrutores de forma a não prejudicar o aten- dimento rotineiro dos usuários da biblioteca.

\section{CONSIDERAÇÕES FINAIS}

Além desse curso, a Biblioteca/CIR da FSP/USP deve oferecer outras formas de instrução em seu Programa Educativo, como sugeriram os participantes do curso Medline e Lilacs. A biblioteca acerta quando oferece níveis diferenciados de orientação, de acordo com a necessidade de informação de cada usuário.

Além da capacitação na busca da informação, o usuário deve também aprender a localizar, selecionar e utilizar a ferramenta mais adequada para que tenha sucesso em sua busca. Esses cursos devem ter como objetivos: capacitar o usuário a desenvolver busca na sua área; selecionar e localizar a melhor forma de obter sua informação; conhecer as várias formas de acesso à informação e ao documen- to; mantê-lo constantemente atualizado nessas tecnologias e formas de acesso.

Não se deve desconsiderar, em um programa educativo, a importância da formação de seus instrutores. O Programa deve promover formação de novos instrutores, contemplar atualização permanente da equipe e, com isso, incentivar a especialização de seu profissional bibliotecário. 


\section{REFERÊNCIAS BIBLIOGRÁFICAS}

1. ALLEN, G. CD-ROM training:wath do the patrons word? Reference Quarterly, v.30, p.88-93, 1990.

2. BRADIGAN, P.S.; MULARSKI, C.A. End user searching in a medical school curriculum: an evaluated modular approach. Bulletin of the Medical Library Association, Chicago, v.74, p.348-53, 1989.

3. CRELLIN, J. Implications of CD-ROMs for reference services in university libraries. Library Management, v.13, n.2, p.208, 1992

4. CUENCA, A M. B. Usuário da busca informatizada: avaliação do Curso Medline/ Lilacs no contexto acadêmico. São Paulo, 1997. [Dissertação de Mestrado Faculdade de Saúde Pública da USP]

5. EARL, M.F. Medical students as CD-ROM end-user trainers. Bulletin of the Medical Library Association, v.79, p.657,1991 .

6. FISHER, J.; BJORNER, S. Enabling online end-user searching: an expanding role for librarians. Special Libraries, v.85, p.281-91, 1994.

7. HEPWORTH, J.B. Training staff and endusers for automated retrieval systems in the health sciences. Education in Information, v.9, p. 97-106, 1991.

\section{Lilacs bases: Evaluation of} content, structure and methodology

\section{Abstract}

This study analyses the results of end-user instruction through the databases modulated course in cd-rom Medline/ Lilacs, modality of the Library Educational Program of the Faculdade de Saúde Pública da Universidade de São Paulo, offered to health sciences scientists and postgraduate students. The whole of the category studied was 92 end-users, between November/1993 and November/ 1995. This study also analyses the Course contents, structure, methodology and didactic materials. This paper gets to the conclusion that end-users, in the whole, consider to be more important that the Library maintains this course and this kind of information user continuous educational program. This survey findings suggests to the Course.

\section{Key words}

Computer user training; End user searching; End user training; Medline and Lilacs modulated course; End user structure; Metodology and contents course.
8. LAMBERT, J. Managing CD-ROM services Librarianship Information Science, v.26, p.23-8, 1994.

9. LUDWIG, L; MIXTER,J.K.; EMANUELE, M.A. Uses attitudes toward end user literature searching. Bulletin of the Medical Library Association, v.76, p.7-13, 1988.

10. MAXYMUK, J. Considerations for CD-ROM instruction. $C D$-ROM Professional, v.4, p.47-9, 1991

11. MUELLER, M.H. ; FOREMAN, G. Library instruction for medical students during a curriculum elective. Bulletin of the Medical Library Association, v.75, p.2536, 1987.

12. MULARSKI, C.A. ; BRADIGAN, P.S. End user searching review of a modular program. Bulletin of the Medical Library Association, v.81, p.61-3,1993.

13. POISSON, E.H. End user searching in medicine. Bulletin of the Medical Library Association, v.74, p.293-9, 1986.

14. REESE, T. CD-ROM end-user instruction: issues and challenges. Microcomputer Information Management, v.10, p. 13154, 1993. in academic libraries. Journal of the
15. RING, D.M. ; MEER, P.F.V. Designing a computerized instructional training room for the library. Special Libraries, v.85, p.15461, 1994.

16. ROWLEY, J. Informática para bibliotecas. Brasilia, Briquet de Lemos Livr., 1994.

17. SOLLENBERGER, J. ; Smith, B.T. Teaching computer searching to health care profissionals: why it take so long? Medical Reference Services Quarterly, v.6, n.4, p.45-51, 1987.

18. STARR, S.S. ; RENFORD, B.L. Evaluation of a program to teach health profissionals to search Medline. Bulletin of the Medical Library Association, v.75, p.193-201, 1987.

\section{Angela Maria Belloni Cuenca}

\section{Maria do Carmo Avamilano Alvarez}

\section{Maria Lucia Evangelista de Faria Ferraz}

\section{Eidi Raquel Franco Abdalla}

Bibliotecárias instrutoras do Curso Medline/ Lilacs do Programa Educativo da Biblioteca: Centro de Referência em Saúde Pública (CIR) da Faculdade de Saúde Pública da USP.

E.mail abcuenca@usp.br 